\title{
Warfarin in Systemic Sclerosis-associated and Idiopathic Pulmonary Arterial Hypertension. A Bayesian Approach to Evaluating Treatment for Uncommon Disease
}

\author{
SINDHU R. JOHNSON, JOHN T. GRANTON, GEORGE A. TOMLINSON, HADDAS A. GROSBEIN, THAOLAN LE, \\ PETER LEE, M. ELIZABETH SEARY, GILLIAN A. HAWKER, and BRIAN M. FELDMAN
}

\begin{abstract}
Objective. Warfarin is recommended in systemic sclerosis-associated pulmonary arterial hypertension (SSc-PAH) and idiopathic PAH (IPAH) to improve survival. There is no evidence to support this in $\mathrm{SSc}-\mathrm{PAH}$ and the evidence in IPAH is conflicting. We evaluated the ability of warfarin to improve survival using 2 large SSc-PAH and IPAH cohorts.

Methods. The effect of warfarin on all-cause mortality was evaluated. Bayesian propensity scores (PS) were used to adjust for baseline differences between patients exposed and not exposed to warfarin, and to assemble a matched cohort. Bayesian Cox proportional hazards models were constructed using informative priors based on international PAH expert elicitation.

Results. Review of 1138 charts identified 275 patients with SSc-PAH ( $\mathrm{n}=78 ; 28 \%$ treated with warfarin) and 155 patients with IPAH $(n=91 ; 59 \%$ treated with warfarin). Baseline differences in PAH severity and medications were resolved using PS matching. In the matched cohort of 98 patients with SSc-PAH (49 treated with warfarin), the posterior median hazard ratio (HR) was 1.06 [95\% credible interval (CrI) $0.70,1.63$ ]. In the matched cohort of 66 patients with IPAH (33 treated with warfarin), the posterior median $\mathrm{HR}$ was $1.07(95 \% \mathrm{CrI} 0.57,1.98)$. The probability that warfarin improves median survival by 6 months or more is $23.5 \%$ in SSc-PAH and $27.7 \%$ in IPAH. Conversely, there is a > $70 \%$ probability that warfarin provides no significant benefit or is harmful.

Conclusion. There is a low probability that warfarin improves survival in SSc-PAH and IPAH. Given the availability of other PAH therapies with demonstrable benefits, there is little reason to use warfarin to improve survival for these patients. (First Release Jan 15 2012; J Rheumatol 2012;39:276-85; doi:10.3899/jrheum.110765)
\end{abstract}

Key Indexing Terms:

BAYESIAN

CONFOUNDING

PROPENSITY SCORE
SCLERODERMA

\section{PULMONARY HYPERTENSION}

WARFARIN
From the Division of Rheumatology, Department of Medicine, Toronto Western Hospital; Department of Health Policy, Management and Evaluation, and the Dalla Lana School of Public Health, University of Toronto; Divisions of Respirology and Critical Care Medicine, Department of Medicine, University Health Network; Division of Clinical Decision Making and Health Care, Toronto General Research Institute; Division of Rheumatology, Department of Paediatrics, The Hospital for Sick Children; Division of Rheumatology, Department of Medicine, Mount Sinai Hospital; and Division of Rheumatology, Department of Medicine, Women's College Hospital, Toronto, Ontario, Canada.

Supported by operating grants from the Canadian Institutes of Health Research and the Scleroderma Society of Ontario. Dr. Johnson has been awarded a Canadian Institutes of Health Research Clinician Scientist Award and Abbott Scholar Award in Rheumatology Research. Dr. Hawker is supported as the F.M. Hill Chair in Academic Women's Medicine, and a Distinguished Senior Rheumatologist Researcher of The Arthritis Society, University of Toronto. Dr. Feldman holds a Canada Research Chair in Childhood Arthritis.

S.R. Johnson, MD, Division of Rheumatology, Department of Medicine, Toronto Western Hospital, Department of Health Policy, Management and Evaluation, University of Toronto, Division of Rheumatology, Department of Medicine, Mount Sinai Hospital; J.T. Granton, MD, Divisions of Respirology and Critical Care Medicine, Department of
Medicine, University Health Network; G.A. Tomlinson, PhD, Department of Health Policy, Management and Evaluation and Dalla Lana School of Public Health, University of Toronto, and Division of Clinical Decision Making and Health Care, Toronto General Research Institute; H.A. Grosbein, BSc, Division of Rheumatology, Department of Paediatrics, The Hospital for Sick Children; T. Le, BSc, Divisions of Respirology and Critical Care Medicine, Department of Medicine, University Health Network; P. Lee, MD, Division of Rheumatology, Department of Medicine, Mount Sinai Hospital; M.E. Seary, BSc, Division of Rheumatology, Department of Paediatrics, The Hospital for Sick Children; G.A. Hawker, MD, MSc, Department of Health Policy, Management and Evaluation, University of Toronto, and Division of Rheumatology, Department of Medicine, Women's College Hospital; B.M. Feldman, MD, MSc, Department of Health Policy, Management and Evaluation and Dalla Lana School of Public Health, University of Toronto, Division of Rheumatology, Department of Paediatrics, The Hospital for Sick Children.

Address correspondence to Dr. S. Johnson, Division of Rheumatology, Ground Floor, East Wing, Toronto Western Hospital, 399 Bathurst Street, Toronto, Ontario M5T 2S8, Canada.E-mail: Sindhu.Johnson@uhn.on.ca Full Release Article. For details see Reprints/Permissions at jrheum.org Accepted for publication September 15, 2011. 
The evaluation of therapy in uncommon disease involves many challenges. Problems stem from the small numbers of patients available for participation in randomized controlled trials (RCT), and the cost of a multicenter trial (which may be the only way to accrue enough subjects) can be insurmountable ${ }^{1}$. Further, there is limited availability of funding for evaluation of older therapies. So some necessary RCT will never be undertaken. Researchers have been challenged to develop methods using observational data to obtain unbiased estimates of treatment effect comparable to those that would be obtained in $\mathrm{RCT}^{2}$. Recently, innovative methodologies and improved computational ability have resulted in the development of strategies that can give such estimates of treatment effect.

An example of providing evidence upon which to base therapy for an uncommon disease, and an older therapy for which funding for research is scarce, is the use of warfarin for improving survival in pulmonary arterial hypertension (PAH). PAH is a lethal disease characterized by elevated pulmonary artery pressure that leads to dyspnea, heart failure, and death. In the setting of systemic sclerosis (SSc), the prevalence of $\mathrm{PAH}$ ranges from $5 \%$ to $12 \% \%^{3,4,5}$, and is a leading cause of death ${ }^{6,7}$. Historically, SSc-PAH had a median survival of 12 months $^{7}$. In the modern treatment era, the median survival has improved to 3-4 years ${ }^{4,8}$. Idiopathic pulmonary arterial hypertension (IPAH) has an incidence ranging from 2.4 to 7.6 cases per million population, and a prevalence ranging from 6.5 to 25 cases per million ${ }^{9}$. Untreated, IPAH has a median survival of 2.8 years ${ }^{10}$. In the modern treatment era, 3-year survival has improved to $76 \%$ to $85 \% \%^{11,12}$. One inexpensive and readily available potential treatment is warfarin. Anticoagulation therapy for patients with PAH has been recommended with the rationale that PAH may be the result of thrombotic arteriopathy and abnormalities in the coagulation cascade ${ }^{13,14,15}$. Our systematic review of the literature found that the evidence to support this recommendation is limited by methodological constraints and conflicting studies ${ }^{16}$. Five studies support the effect of anticoagulation in $\operatorname{IPAH}^{17,18,19,20,21}$, while 3 studies do $\operatorname{not}^{22,23,24,25}$. None of the studies were placebo-controlled or blinded. Only 1 of the 8 studies was prospective. Most significantly, none of the studies were randomized. The major threat to the validity of the results of these studies is confounding by indication. In addition, these studies were limited by small sample sizes, and thus the negative results may reflect insufficient power.

The role of warfarin in the treatment of SScPAH has not been established. There are no studies evaluating the effect of warfarin in this population. The recommendation to consider warfarin in SSc-PAH is based on expert opinion and potential benefits generalized from IPAH studies ${ }^{26,27}$. However, the theoretical benefits of warfarin may be offset by an increased risk of gastrointestinal (GI) bleeding and stroke, which can lead to hospitalization, morbidity, and mortality. Warfarin is associated with "major bleeding" rates ranging from $2 \%$ to $3 \%$ annually $^{28,29}$. Further, SSc patients with luminal telangiectasia or gastric antral vascular ectasia (GAVE) may be at higher risk of GI bleeding ${ }^{30,31}$. An evaluation of the benefits and risks of warfarin in SSc-PAH and IPAH is needed. Newer therapies for PAH, such as prostacyclin analogs, endothelin receptor antagonists, and phosphodiesterase inhibitors, have been shown to have beneficial effects on exercise capacity and dyspnea ${ }^{32,33}$. However, they are very expensive, logistically difficult to administer, and not equally accessible to patients. In the era of modern $\mathrm{PAH}$ management, it is unlikely that a trial evaluating the effect of warfarin will be conducted. Yet it remains uncertain whether warfarin is effective, and uncertain whether it has a place in modern PAH therapy.

This uncertainty is reflected in current guidelines. McLaughlin, et al recommended anticoagulation in SSc-PAH with advanced disease and without contraindications, and recommended anticoagulation in IPAH - based on committee consensus ${ }^{27}$. Galie, et al recommended that anticoagulation be considered in IPAH and that it may be considered in SSc-PAH (Class IIb recommendation: usefulness/efficacy is less established by evidence/opinion $)^{26}$. Barst, et al gave no recommendation for the use of warfarin in SSc-PAH and gave only a "moderate recommendation on the basis of expert opinion" for warfarin use in IPAH ${ }^{14}$.

Our objectives were to evaluate the effect of warfarin on survival in patients with SSc-PAH and IPAH, respectively. We used state of the art methods to obtain estimates of treatment effect comparable to those that would have been obtained from a randomized trial.

\section{MATERIALS AND METHODS}

Patients. The Toronto Scleroderma Program and the University Health Network Pulmonary Hypertension Programme are the largest published longitudinal cohorts of their kind in Canada. Patients were included if they had a diagnosis of SSc with PAH or IPAH defined as a mean pulmonary artery pressure (mPAP) $>25 \mathrm{~mm} \mathrm{Hg}$ and pulmonary capillary wedge pressure $(\mathrm{PCWP})<15 \mathrm{~mm} \mathrm{Hg}$ by cardiac catheterization ${ }^{34}$, and age $>17$ years. Patients were excluded if they had another etiology for pulmonary hypertension [human immunodeficiency virus, anorexigen use, portal hypertension, cardiac abnormalities (left heart disease such as systolic, diastolic dysfunction or valvular dysfunction)], interstitial lung disease (forced vital capacity $<70 \%$ predicted and bibasilar reticular abnormalities with minimal ground-glass on high-resolution computerized tomography of the thorax or thromboembolic disease), a diagnosis of other connective tissue disease, other indications for warfarin use (atrial fibrillation, artificial heart valve, pulmonary thromboembolic disease), or any contraindications to warfarin use.

Exposure. The exposure was treatment with warfarin at any time after diagnosis of PAH. A minimum duration of exposure or minimum dose was not specified.

Outcome. The primary outcome was time from diagnosis of PAH to death from all causes. Patients who were alive at the end of the followup were censored. Dates of death were obtained from the clinic chart, hospital electronic record, or obituary. Online obituary websites were searched to identify patients who had died. The date of death from the obituary was used if there was a correct match on first and last name, sex, city/town, and use of

Personal non-commercial use only. The Journal of Rheumatology Copyright @ 2012 . All rights reserved. 
the terms "scleroderma" or "pulmonary hypertension" in the obituary text. If a patient was alive for the last scheduled clinic visit, or survival status on January 1, 2008, was unknown, the family/referring physicians were contacted using a standardized letter that was faxed and mailed twice, and up to 2 subsequent telephone calls. Information about survival status, cause of death, or date last seen was collected.

Data collection. Data were abstracted by a single abstractor from charts, standardized research protocols, and hospital electronic records using a standardized abstraction form. Data included date of birth, sex, postal code, dates of SSc and PAH diagnosis, etiology of PAH, comorbidities, treatments, adverse events with warfarin, baseline functional class, cardiac hemodynamics [mPAP, PCWP, cardiac output (CO), mean right atrial pressure (RAP), pulmonary vascular resistance (PVR)], right ventricular measures (size, function, systolic pressure), and pulmonary function tests. GI bleeding was defined as the presence of upper or lower GI bleeding requiring treatment with 1 or more units of blood.

Data administration. Data collected from research protocols were verified against the chart or hospital record. Data were double-entered into a Filemaker Pro version 8.5 database. Data entry errors were minimized through the use of automatic value ranges and internal logic checks.

Analytic overview. Propensity score (PS) methods were used to create a cohort of matched treated and untreated patients, as a method of bias reduction. A fully Bayesian analysis was undertaken using a survival outcome. This approach takes into account preexisting knowledge regarding the effect of warfarin on survival expressed as prior probability distributions ("priors"). The result is a posterior probability distribution that allows for inferences about treatment effect to be made using probability statements, and that takes into consideration both this preexisting knowledge and new data, and allows for inferences about treatment effect to be made using probability statements.

PS evaluation. A Bayesian logistic regression model was fitted using baseline variables and exposure to warfarin as the outcome variable. Variables were chosen based on elicitation from international PAH experts and clinical sensibility ${ }^{35}$. Variables that PAH experts reported as important included sex, disease subtype, PAP, right heart size and function, functional class, disease duration, comorbidities, and concomitant medications ${ }^{35}$. Uninformative priors were specified with a diffuse normal distribution, mean of 0 and precision of $10^{-6}$. For each patient, the median posterior probability of exposure was used as the PS. Treated and untreated patients were matched without replacement on functional class and PS, using 1:1, nearest-neighbor matching with a caliper width of $0.2 \mathrm{SD}$ of the PS. The goodness-of-fit of the PS was evaluated by the degree to which it resulted in balance of baseline variables between the warfarin-exposed patients and the nonexposed patients. A method of assessing balance is evaluation of the standardized difference. Equations show the standardized differences for binary and continuous variables.

$$
d=\frac{\left(\hat{p}_{\text {treatment }}-\hat{p}_{\text {control }}\right)}{\frac{\sqrt{\hat{p}_{\text {treatment }}\left(1-\hat{p}_{\text {treatment }}\right)+\hat{p}_{\text {control }}\left(1-\hat{p}_{\text {control }}\right)}}{2}}
$$

Standardized differences for comparing dichotomous variables:

$\mathrm{d}=$ standardized difference

$\mathrm{p}=$ prevalence of baseline characteristic

$$
d=\frac{\left(\bar{x}_{\text {treatment }}-\bar{x}_{\text {control }}\right)}{\frac{\sqrt{S_{\text {treatment }}^{2}+S_{\text {control }}^{2}}}{2}}
$$

Standardized difference for comparing means:

$\mathrm{d}=$ standardized difference

$\mathrm{x}=$ mean of baseline characteristic

$\mathrm{s}^{2}=$ variance of baseline characteristic
As in a randomized trial, there may be some residual imbalance in baseline characteristics in the matched sample. It has been recommended that the threshold for acceptable residual imbalance is the residual imbalance that would be observed in a similar size clinical trial in the same clinical area. Alternatively, it has been recommended that an absolute difference of $10 \%$ or less is considered a good match $^{36}$.

Priors. Priors are quantified expressions of knowledge and/or beliefs regarding a treatment effect preceding the conduct of the study. A variety of priors were used to evaluate the sensitivity of the analysis to a range of viewpoints that should be considered when interpreting the evidence ${ }^{37}$. An uninformative prior was used to evaluate the data, independent of preexisting knowledge. For SSc-PAH and IPAH separately, the uninformative prior was specified for the log-hazard ratio (HR) comparing the 2 groups: this was a diffuse normal distribution with a mean of 0 , and a precision of $10^{-6}$. An informative prior based on our study of international PAH experts' beliefs about the effect of warfarin on survival, and an optimistic prior using information from the $10 \%$ of experts specifying the smallest HR were constructed as follows ${ }^{35}$. Each expert's distribution for the probability of 3 -year survival with warfarin treatment was combined with the expert's probability of 3-year survival without warfarin treatment to generate the expert's prior distribution for the $\log (\mathrm{HR})$. The distributions were averaged across experts to generate the group mean prior. The SSc-PAH group prior for the $\log (\mathrm{HR})$ was best represented by a Student $\mathrm{t}$ distribution with 4 degrees of freedom, a mean of 0.03 , and SD of 0.27 . The SSc-PAH optimistic prior was best represented by a Student $t$ distribution with 3 degrees of freedom, a mean of -0.15 , and SD of 0.23 . The IPAH group prior was best represented by a Student t distribution with 5 degrees of freedom, a mean of 0.09 , and SD of 0.35 . The IPAH optimistic prior was best represented by a Student $t$ distribution with 4 degrees of freedom, a mean of -0.08 , and SD of 0.29 . Box's measure of conflict was used as a method to compare the experts' informative prior with the data. It is analogous to a traditional $\mathrm{p}$ value in measuring the predictive probability of getting a result at least as extreme as that observed ${ }^{37}$.

It has been recommended that Bayesian analyses include a tri-plot. A tri-plot is used to illustrate the prior and the new data expressed as a likelihood function. It also illustrates the posterior probability distribution, that is, how the prior probability distribution has changed with the incorporation of new data.

Survival model. Patients who were alive on January 1, 2008, were right censored. Median survival and survival probabilities in the treated and untreated patients in the matched cohort were determined using Kaplan-Meier survival curves. Within the matched pairs, we used a matched Cox proportional hazards model to estimate the effect of warfarin on all-cause mortality.

For SSc-PAH and IPAH separately, we evaluated the probability that warfarin improves median survival by 6 months or more. A timepoint was necessary for us to make a direct probability statement. We chose 6 months or more, as we believe that this would be an improvement in survival that would be considered clinically important by both patients and clinicians. We assumed a constant hazard rate (exponential survival) in the patients not exposed to warfarin and multiplied this by samples from the posterior distribution of the HR for warfarin to obtain samples of the hazard rate in the exposed. We chose a constant (exponential) hazard when computing the probability that there was a survival benefit $>6$ months for computational simplicity and because the exponential is widely used in the modeling of survival. A simple calculation converts the 2 hazard rates to a difference in median survival between the 2 groups. We computed the proportion of samples where the difference in median survival time exceeded 6 months.

Simulation. For SSc-PAH and IPAH separately, we assessed whether our PS-matched data gave comparable differences in baseline characteristics to that observed in a randomized trial of the same size. In this simulation, samples of size 98 (the size of the matched SSc-PAH cohort) were drawn from the observed SSc-PAH cohort of 275 patients and randomized in a 1:1 ratio to treatment or control. For each baseline characteristic, the absolute standardized difference was calculated. This was repeated 10,000 times, 
and we counted the number of times a standardized difference between the 2 groups exceeded the observed value in the PS-matched sample. For the patients with IPAH, we carried out a similar procedure, drawing samples of size 66 from the observed IPAH cohort of 155 patients.

All Bayesian analyses used a burn-in of 5000 followed by collection of 10,000 Monte Carlo Markov chain (MCMC) updates. The HR, 95\% credible interval, and probabilities were computed from these MCMC samples from the posterior distribution. Where appropriate, convergence was evaluated using the Brooks-Gelman-Rubin convergence statistic. The reporting of the analysis and results are in accord with the ROBUST criteria ${ }^{38}$. Analyses were performed using SAS (v9.2, SAS Institute, Cary, NC, USA), R (v2.8.1, The R Foundation for Statistical Computing), and WinBUGS (v1.4.3, Imperial College and Medical Research Council, London, UK).

Research ethics board approval was obtained prior to the our study.

\section{RESULTS}

Patients. A review of 1138 charts identified 275 patients with SSc-PAH. Seventy-eight (28\%) of them received warfarin. There were 155 patients identified with IPAH; 91 $(59 \%)$ of them received warfarin. Warfarin-treated patients had worse baseline measures of PAH severity and more use of PAH medications (Tables 1 and 2). Calcium channel blocker (CCB) use was more frequent among patients with SSc-PAH than patients with IPAH. This is likely due to the frequent use of CCB for the treatment of Raynaud's phenomenon. There were no significant differences in CCB use between the warfarin-exposed and nonexposed patients in the SSc-PAH and IPAH matched cohorts, respectively.

GI bleeding occurred in $7 \%(13 / 197)$ of the warfarin-unexposed and $8 \%(8 / 78)$ of the warfarin-exposed patients with SSc-PAH. Hemorrhagic stroke occurred in 1 warfarin-unexposed and 1 exposed patient with SSc-PAH. GI bleeding occurred in $2 \%(1 / 64)$ of the warfarin-unexposed and 7\% (6/91) of the warfarin-exposed patients with IPAH. Hemorrhagic stroke occurred in 1 warfarin-unexposed patient with IPAH.

PS matching. The differences in baseline characteristics between the treated and untreated groups were substantially reduced in the PS-matched SSc-PAH and IPAH cohorts (Tables 1 and 2). The largest standardized difference in the matched cohort was 0.27. In each of the SSc-PAH-matched and IPAH-matched cohorts, a standardized difference greater than the recommended 0.10 was observed twice ${ }^{36}$. Our simulation found that in an RCT with 49 patients with SSc-PAH per group and 16 baseline characteristics, there is a $91 \%$ probability of observing an absolute standardized difference of 0.27 or greater, and a $100 \%$ probability of observing at least 2 baseline characteristics with an absolute standardized difference of 0.10 or more. In an RCT with 33 patients with IPAH per group and 16 baseline characteristics, there is a $99.9 \%$ probability of observing an absolute standardized difference of 0.20 or greater, and a $100 \%$ probability of observing at least 2 baseline characteristics with an absolute standardized difference of 0.10 or more. Therefore, both PS-matched cohorts had differences in baseline covariates smaller than those that would be observed in an RCT of the same size.

We also evaluated differences in baseline hemodynamics not included in the PS model between warfarin-unexposed

Table 1. Characteristics of patients with SScPAH.

\begin{tabular}{|c|c|c|c|c|c|c|}
\hline \multirow[t]{2}{*}{ Characteristics } & \multicolumn{2}{|c|}{$\begin{array}{l}\text { Unmatched, } \\
\mathrm{n}=275\end{array}$} & \multicolumn{2}{|c|}{$\begin{array}{l}\text { Matched, } \\
\mathrm{n}=98\end{array}$} & \multicolumn{2}{|c|}{$\begin{array}{c}\text { Absolute } \\
\text { Standardized Difference }\end{array}$} \\
\hline & $\begin{array}{c}\text { No Warfarin, } \\
n=197\end{array}$ & $\begin{array}{l}\text { Warfarin, } \\
\mathrm{n}=78\end{array}$ & $\begin{array}{c}\text { No Warfarin, } \\
n=49\end{array}$ & $\begin{array}{l}\text { Warfarin, } \\
n=49\end{array}$ & $\begin{array}{l}\text { Unmatched } \\
\mathrm{n}=275\end{array}$ & $\begin{array}{c}\text { Matched } \\
\mathrm{n}=98\end{array}$ \\
\hline Female sex, n (\%) & $165(84)$ & $66(85)$ & $45(92)$ & $44(90)$ & 0.22 & 0.07 \\
\hline \multicolumn{7}{|l|}{ PAH characteristics at diagnosis } \\
\hline mPAP mm Hg, mean (SD) & $39.0(14.3)$ & $46.8(14.3)$ & $38.8(15.3)$ & $42.5(11.8)$ & 0.54 & 0.27 \\
\hline Moderate-severe RV hypokinesis, n (\%) & $25(13)$ & $25(32)$ & $11(22)$ & $13(27)$ & 0.22 & 0.05 \\
\hline \multicolumn{7}{|l|}{ Comorbidities, n (\%) } \\
\hline Cancer & $21(11)$ & $8(10)$ & $7(14)$ & $6(12)$ & $<0.01$ & 0.02 \\
\hline Coronary artery disease & $27(14)$ & $7(9)$ & $6(12)$ & $5(10)$ & 0.05 & 0.02 \\
\hline Diabetes mellitus & $10(5)$ & $7(9)$ & $4(8)$ & $2(4)$ & 0.04 & 0.04 \\
\hline Hyperlipidemia & $11(6)$ & $9(12)$ & $6(12)$ & $3(6)$ & 0.06 & 0.06 \\
\hline \multicolumn{7}{|l|}{ Concomitant medications, $\mathrm{n}(\%)$} \\
\hline Calcium channel blocker & $104(53)$ & $33(42)$ & $23(47)$ & $23(47)$ & 0.14 & 0 \\
\hline ER antagonist & $35(18)$ & $33(42)$ & $9(18)$ & $15(31)$ & 0.29 & 0.14 \\
\hline PDE inhibitor & $16(8)$ & $5(6)$ & $3(6)$ & $5(10)$ & 0.02 & 0.04 \\
\hline Prostaglandin analog & $10(5)$ & $13(17)$ & $4(8)$ & $4(8)$ & 0.12 & 0 \\
\hline
\end{tabular}

SScPAH: systemic sclerosis-associated pulmonary arterial hypertension; mPAP: mean pulmonary artery pressure; WHO: World Health Organization; $\mathrm{RV}$ : right ventricular; ER: endothelin receptor; PDE: phosphodiesterase.

Personal non-commercial use only. The Journal of Rheumatology Copyright (c) 2012. All rights reserved. 
Table 2. Characteristics of patients with idiopathic pulmonary arterial hypertension (IPAH).

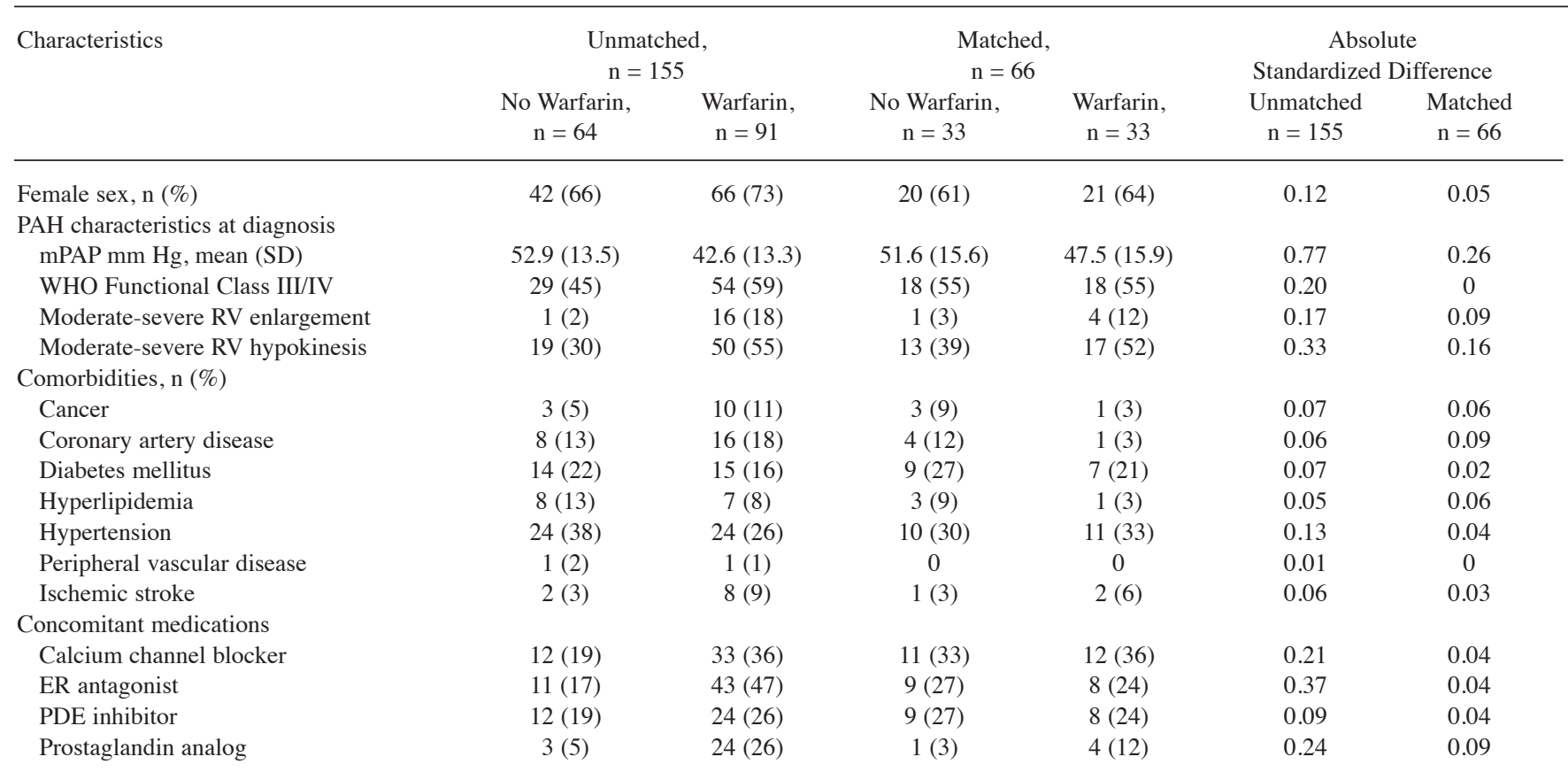

mPAP: mean pulmonary artery pressure; RV: right ventricular; WHO: World Health Organization; RV: right ventricular; ER: endothelin receptor; PDE: phosphodiesterase.

and exposed patients. In the SSc-PAH matched cohort, the warfarin-unexposed patients compared to the warfarin-exposed patients had a mean (SD) RAP 12.8 (4.6) $\mathrm{mm}$ $\mathrm{Hg}$ versus 10.2 (7.6) $\mathrm{mm} \mathrm{Hg}, \mathrm{CO} 3.5$ (0.8) $\mathrm{l} /$ min versus 3.4 (1.7) $\mathrm{l} / \mathrm{min}$, PCWP 14.2 (8.13) $\mathrm{mm} \mathrm{Hg}$ versus 9.8 (4.5) $\mathrm{mm}$ $\mathrm{Hg}$, and PVR 492 (564) dyn $\bullet \bullet \mathrm{cm}^{-5}$ versus 528 (346) dyn $\bullet \mathrm{cm}^{-5}$. In the matched cohort of patients with IPAH, the warfarin-unexposed patients compared to warfarin-exposed patients had a mean RAP 14.9 (9.0) $\mathrm{mm} \mathrm{Hg}$ versus 14.6 (8.7) $\mathrm{mm} \mathrm{Hg}, \mathrm{CO} 27.9$ (13.5) $1 /$ min versus 27.0 (8.7) $1 / \mathrm{min}$, PCWP 10.5 (4.2) $\mathrm{mm} \mathrm{Hg}$ versus 9.4 (4.5) $\mathrm{mm}$ $\mathrm{Hg}$, and PVR 1278 (502) dyn॰s॰cm${ }^{-5}$ versus 923 (538) dyn $\bullet \mathrm{s}^{\bullet} \mathrm{cm}^{-5}$.

Survival. The 3-year survival in the matched SSc-PAH cohort was $61 \%$ for the warfarin-unexposed patients and $58 \%$ for the warfarin-exposed patients. The 3-year survival in the matched IPAH cohort was $83 \%$ for both the warfarin-unexposed and exposed patients. The results of the Cox model survival analysis are presented in Table 3 . The Bayesian tri-plots illustrating the prior, likelihood, and posterior distributions for SSc-PAH and IPAH are presented in Figures 1 and 2, respectively. The tri-plots illustrate that the addition of data from our study have improved the precision (decreased the uncertainty) around the estimated HR. Box's measure of conflict between the group prior and the data was 0.62 for SSc-PAH and 0.91 for IPAH. This indicates no evidence of a significant discrepancy between the prior and the data.

Given exponential survival with a median of 4.9 years in
Table 3. Cox proportional hazards model survival analysis.

\begin{tabular}{lcc}
\hline Statistical Method & Sample Size & $\begin{array}{c}\text { Bayesian Hazard } \\
\text { Ratio*, median } \\
(95 \% \text { CrI })\end{array}$ \\
\hline SScPAH & & \\
Unmatched data & 275 & $1.50(1.02,2.19)$ \\
Matched data, noninformative prior & 98 & $1.09(0.59,2.02)$ \\
Matched data, informative optimistic prior & 98 & $1.06(0.70,1.63)$ \\
Matched data, informative optimistic prior & 98 & $0.94(0.65,1.46)$ \\
IPAH & & \\
Unmatched data & 155 & $1.31(0.63,2.92)$ \\
Matched data, noninformative prior & 66 & $1.01(0.35,3.01)$ \\
Matched data, informative group prior & 66 & $1.07(0.57,1.98)$ \\
Matched data, informative optimistic prior & 66 & $0.94(0.54,1.64)$ \\
\hline
\end{tabular}

\footnotetext{
* Hazard ratio $>1$ indicates increased mortality associated with warfarin exposure. Hazard ratio $<1$ indicates decreased mortality associated with warfarin exposure. CrI: credible interval; SScPAH: scleroderma-associated pulmonary arterial hypertension; IPAH: idiopathic PAH.
}

the untreated patients with SSc-PAH, the probability of improving survival by 6 months or more with warfarin is $23.5 \%$ (i.e., $76.5 \%$ probability of survival worsening, or survival improvement of $<6$ months; Figure 3 ). Given a median survival of 3.9 years in the untreated patients with IPAH, the probability of improving survival by 6 months or more with warfarin is $27.7 \%$ (i.e., $72.3 \%$ probability of survival worsening, or survival improvement of $<6$ months; Figure 4). Sensitivity analyses indicate that if the baseline median survival is as high as 7 years, the probability of improving Personal non-commercial use only. The Journal of Rheumatology Copyright @ 2012 . All rights reserved. 


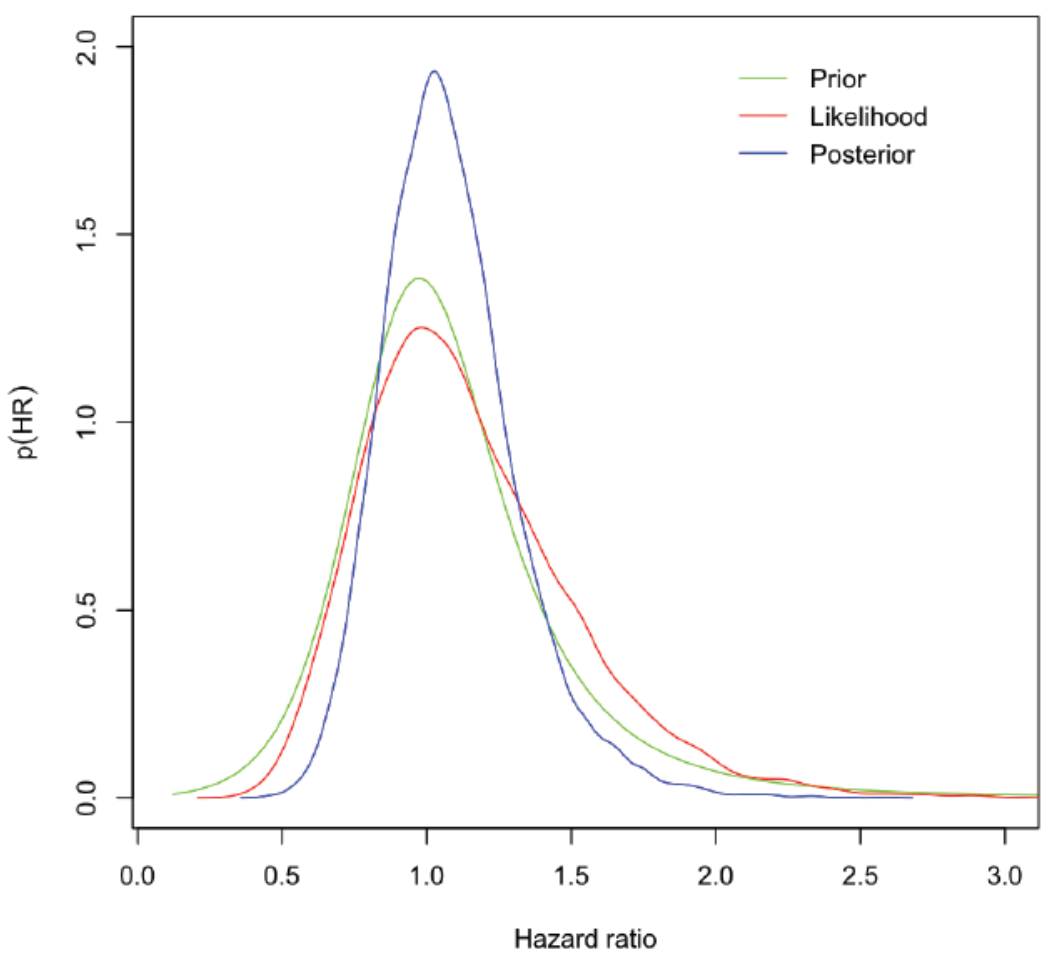

Figure 1. Bayesian tri-plot for effect of warfarin on survival in patients with systemic sclerosis-associated pulmonary arterial hypertension. Hazard ratio $>1$ indicates increased mortality associated with warfarin exposure. Hazard ratio $<1$ indicates decreased mortality associated with warfarin exposure.

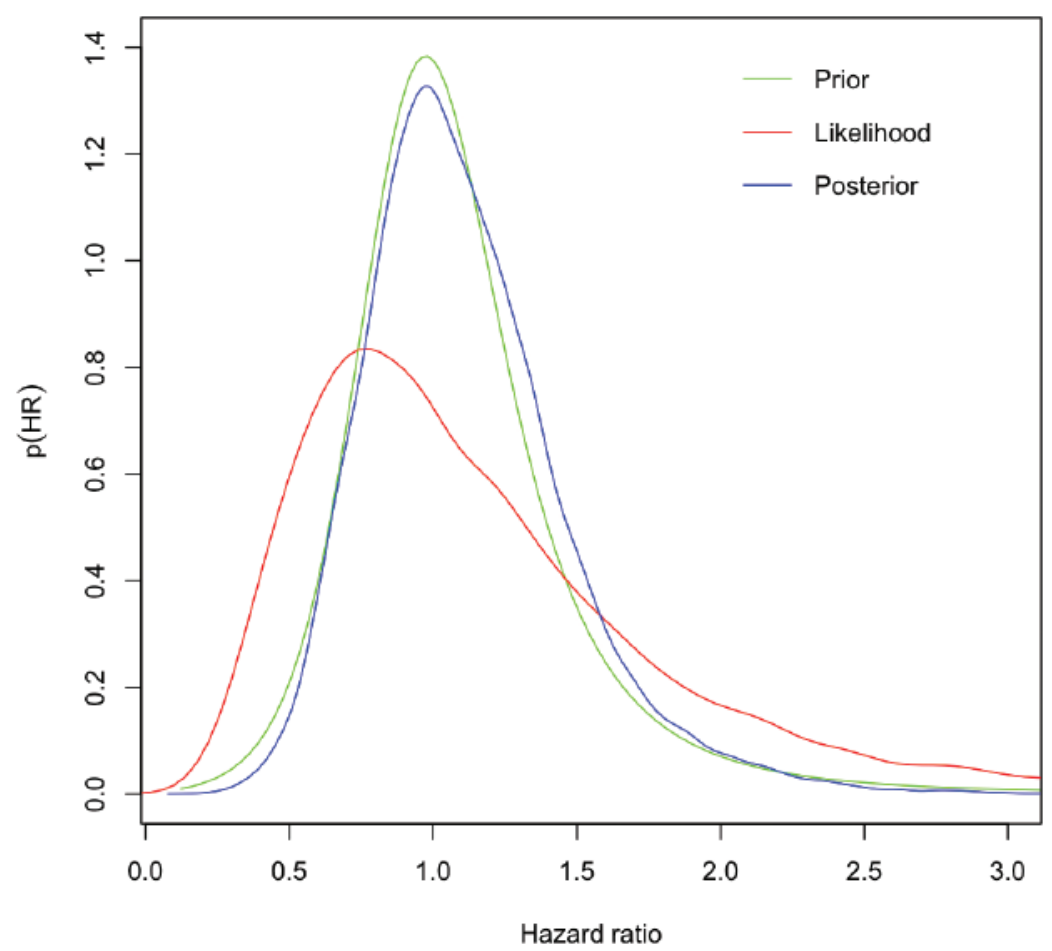

Figure 2. Bayesian tri-plot for effect of warfarin on survival in patients with idiopathic pulmonary arterial hypertension. Hazard ratio $>1$ indicates increased mortality associated with warfarin exposure. Hazard ratio $<1$ indicates decreased mortality associated with warfarin exposure. 


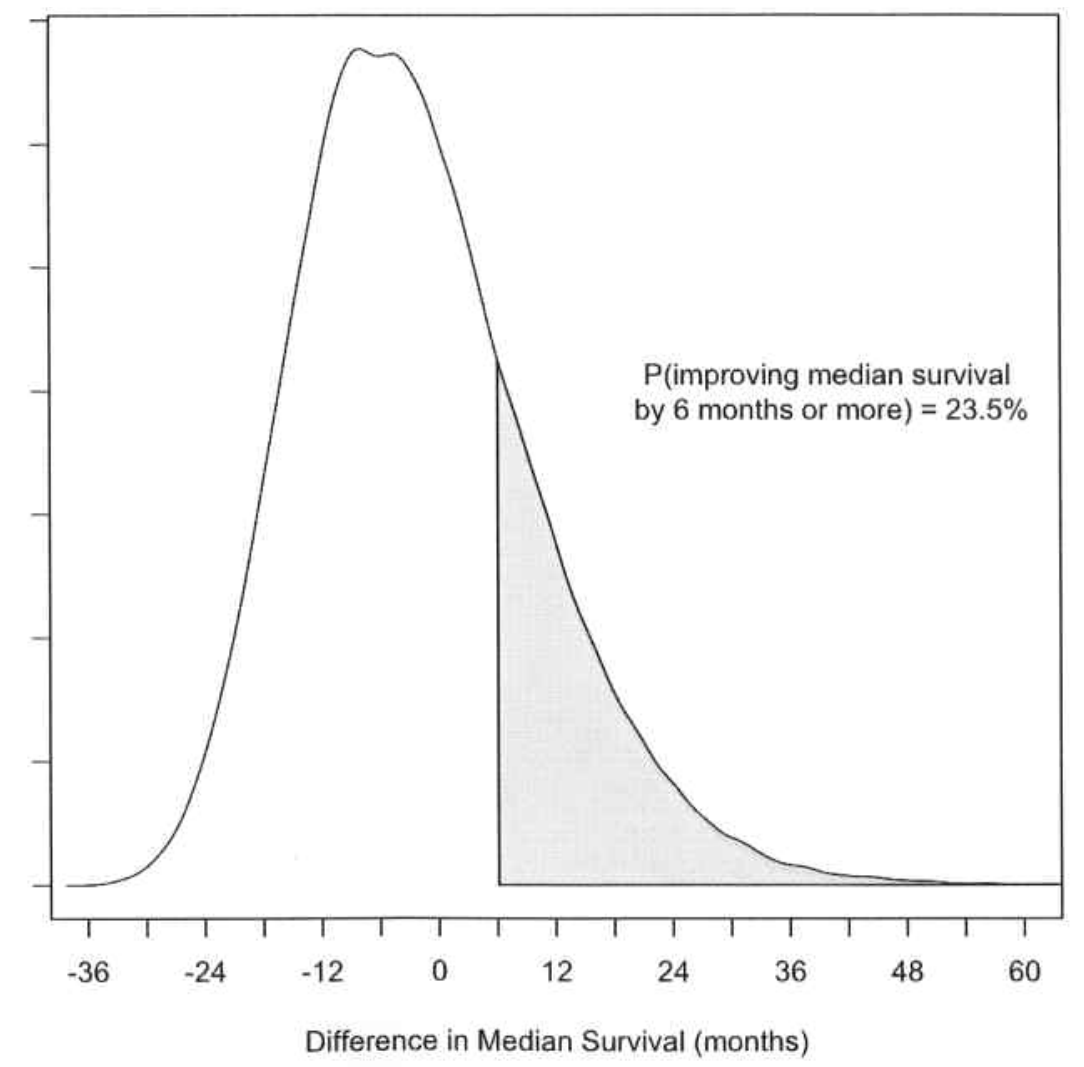

\begin{abstract}
Figure 3. Density plot for difference in median survival times in patients with systemic sclerosis-associated pulmonary arterial hypertension untreated and treated with warfarin, using an informative group prior. Differences in median survival $>0$ indicate improved survival associated with warfarin exposure. Differences in median survival $<0$ indicate worsened survival associated with warfarin exposure. Y-axis indicates relative probability.
\end{abstract}

survival by 6 months or more in either SSc-PAH or IPAH does not exceed $32 \%$.

Using an optimistic prior representing experts with a beneficial view of the effect of warfarin in SSc-PAH, the probability of improving survival by 6 months or more with warfarin is $43 \%$, and does not exceed $50 \%$ if the baseline median survival is as high as 7 years. Using an optimistic prior in IPAH, the probability of improving survival by 6 months or more with warfarin is $41.6 \%$, and does not exceed $50 \%$ if the baseline median survival is as high as 7 years.

\section{DISCUSSION}

With innovative methods, we have used observational data to make estimates of treatment effect comparable to that observed in an RCT. This is a unique methodologic contribution to the literature because the methods we used protect from biases that usually compromise the validity of other retrospective, observational studies. In a setting where a definitive RCT is not feasible because of the large sample size required, cost, and/or political will ${ }^{1}$, this is of great value. Further, we have demonstrated that in both SSc-PAH and IPAH, the probability of a survival benefit with warfarin is low.
We found that both patients with SSc-PAH and patients with IPAH who have been exposed to warfarin have worse functional class, more right ventricular dysfunction, and use more PAH medications than unexposed patients. This suggests that the crude association between warfarin and survival is likely to be confounded (confounding by indication). Failure to account for these systematic differences leads to biased estimates of treatment effect. In this case, it would lead to the conclusion that warfarin worsens survival. This is likely not the case since major hemorrhage was an infrequent cause of death. Our use of PS matching reduced the effect of confounding, allowing us to make a less biased estimate of the treatment effect.

The use of the Bayesian paradigm in the setting of an uncommon disease conferred a number of advantages. First, Bayesian methods allowed us to make direct probability statements about the treatment effect given the data at hand; this contrasts with traditional (frequentist) methods that report on the extremeness of the data (the p value), given an assumption about a true treatment effect ${ }^{39}$. Our inferences were not bound by a decision based on what is usually an arbitrary 0.05 level of significance. This has great utility in the study of uncommon diseases, where numbers of patients

Personal non-commercial use only. The Journal of Rheumatology Copyright $\subset$ 2012. All rights reserved. 


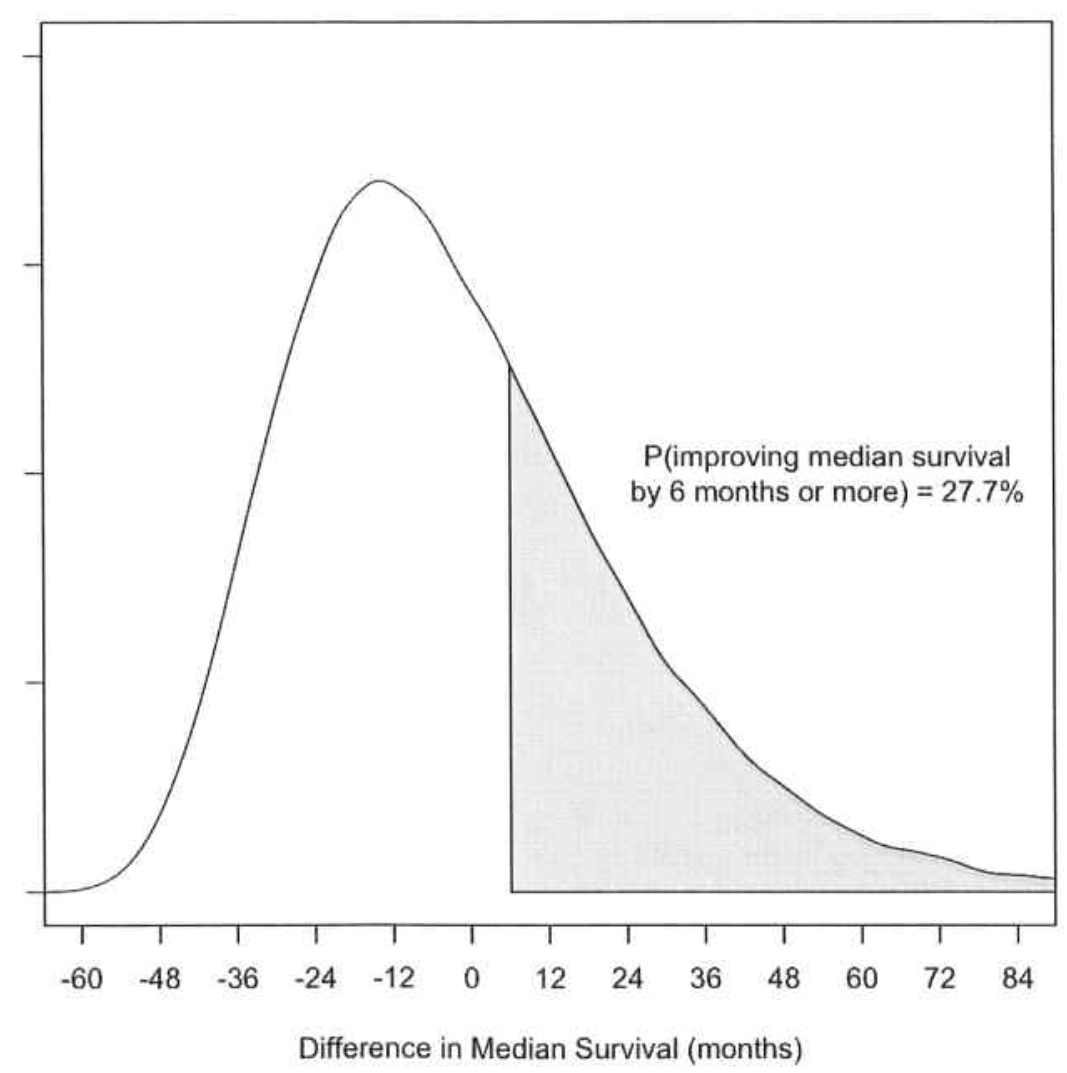

Figure 4. Density plot for difference in median survival times in patients with idiopathic pulmonary arterial hypertension untreated and treated with warfarin, using an informative group prior. Differences in median survival $>0$ indicate improved survival associated with warfarin exposure. Differences in median survival $<0$ indicate worsened survival associated with warfarin exposure. Y-axis indicates relative probability.

available for study (and power to detect a treatment effect at a certain $\mathrm{p}$ value) are limited. Second, in a Bayesian analysis, we are able to compute the probability that a treatment effect is larger than any specified threshold ${ }^{40}$. We are able to make direct evidence-based probability statements that are useful to clinicians. In SSc-PAH and IPAH, there is a low probability of improving survival by 6 months or more with warfarin. Third, Bayesian models allow the analysis to incorporate preexisting knowledge and beliefs in the estimation of a treatment effect, so that the estimate includes all knowledge in the area to date ${ }^{41}$. In our study, evaluation of the matched data with an uninformative prior indicates an HR close to 1. Taking into account international PAH experts' knowledge and experience gives a similar HR, with greater precision. Similarly, we analyzed the data using optimistic priors. Despite using the beliefs of experts who have the most optimistic view about a beneficial treatment effect of warfarin, the probability of improving survival by a median of 6 months or more is $<50 \%$ in either SSc-PAH or IPAH. The use of informative priors reveals how rational individuals can interpret the study findings, given experts' knowledge and experience. Our finding provides a scientific, quantifiable answer to the question, "how should this new piece of evidence change what we currently believe?"37.

We found that treatment with warfarin has a low probability of improving survival in either SSc-PAH or IPAH. To our knowledge, this is the first study to evaluate the effect of warfarin in SSc-PAH. Our findings are discordant with some observational studies of warfarin in IPAH. Our findings could be explained within the context of the evolving understanding of the pathogenesis of PAH over time. The recommendation for warfarin use originated in a time when PAH was believed to be the result of thrombotic arteriopathy and abnormalities in the coagulation cascade ${ }^{15}$. Previous histopathologic studies report a prevalence of thrombotic arteriopathy of $40 \%-57 \% 17,42$. Also, in the past there were limited treatment options. The potential benefit of warfarin outweighed the known risk of hemorrhage. Over the last decade, however, the pathogenesis has been recognized to be much more complex ${ }^{43}$. One potential explanation for the low probability of a beneficial effect of warfarin is that the role of thrombotic arteriopathy in the pathogenesis of PAH may be smaller than previously believed ${ }^{15}$. Therefore, the risks and benefits should be carefully considered when making a decision to use anticoagulation therapy on patients 
with SSc-PAH or IPAH ${ }^{44}$. There is little justification for warfarin in a situation where there is a low probability of survival benefit, and alternative treatment options with demonstrable survival benefit are available.

Our findings also provide interesting insights into adverse events with warfarin in SSc-PAH and IPAH. In our patients with SSc-PAH, there were no differences in the occurrence of hemorrhagic stroke or GI bleeding between warfarin-unexposed and exposed patients. Given the presence of GI vascular lesions (luminal telangiectasia or GAVE), patients with SSc-PAH are potentially at higher risk of GI bleeding. In our study, warfarin use did not increase the risk of major GI bleeding. However, major GI bleeding was defined as necessitating transfusion. Our study did not determine rates of minor bleeding. In the IPAH patients, major GI bleeding occurred more frequently in the warfarin-exposed patients. Hemorrhagic stroke occurred in 1 warfarin-unexposed patient with IPAH.

There are potential limitations that should be considered in the interpretation of our study. The first potential limitation is that our matching did not account for all prognostic factors that are reported in the literature. We included all important confounders that were specified by experts, and our standardized differences showed that our groups were as equal in matching as would be expected in a randomized trial of the same size. However, we could not adjust for unknown confounders. A second limitation is our small study size. In a frequentist analysis we might have low power to detect an important difference. However, in a Bayesian framework, power is not a consideration, and we showed a very low probability of a clinically important difference. A third potential limitation is our categorization of warfarin exposure. Timing of warfarin treatment could affect the outcome. If a patient died before being given warfarin, they would have been classified in the unexposed group. This would bias the results toward a benefit for warfarin. If this is the case, the probability of survival benefit with warfarin in SSc-PAH and IPAH is even less than we report. A fourth potential limitation is our exclusion of patients who had other causes of pulmonary hypertension (e.g., interstitial lung disease). The prognosis of these patients has been shown to be different from patients with $\mathrm{PAH}$, and was not included in this analysis. This affects the generalizability of our study results. Our results apply only to patients with SSc-PAH and IPAH.

The use of innovative methods to make unbiased estimates of treatment effects using observational data is a significant contribution to the study of uncommon diseases. These methods will be valuable to all researchers who are faced with the methodologic challenge of making inferences about treatment effects from observational data of uncommon diseases. In this study, the probability that warfarin improves survival in SSc-PAH and IPAH is low. Given the availability of other PAH therapies with demonstrable bene- ficial effects, there is little usefulness for warfarin in the treatment of SSc-PAH and IPAH.

\section{REFERENCES}

1. Medsger TA Jr. Progressive systemic sclerosis. Clin Rheum Dis 1983;9:655-70.

2. Feinstein AR. Scientific standards in epidemiologic studies of the menace of daily life. Science 1988;242:1257-63.

3. Avouac J, Airo P, Meune C, Beretta L, Dieude P, Caramaschi P, et al. Prevalence of pulmonary hypertension in systemic sclerosis in European Caucasians and metaanalysis of 5 studies. J Rheumatol 2010;37:2290-8.

4. Mukerjee D, St George D, Coleiro B, Knight C, Denton CP, Davar $\mathrm{J}$, et al. Prevalence and outcome in systemic sclerosis associated pulmonary arterial hypertension: Application of a registry approach. Ann Rheum Dis 2003;62:1088-93.

5. Hachulla E, Gressin V, Guillevin L, Carpentier P, Diot E, Sibilia $\mathrm{J}$, et al. Early detection of pulmonary arterial hypertension in systemic sclerosis: A French nationwide prospective multicenter study. Arthritis Rheum 2005;52:3792-800

6. Steen VD, Medsger TA. Changes in causes of death in systemic sclerosis, 1972-2002. Ann Rheum Dis 2007;66:940-4.

7. Koh ET, Lee P, Gladman DD, Abu-Shakra M. Pulmonary hypertension in systemic sclerosis: An analysis of 17 patients. $\mathrm{Br}$ J Rheumatol 1996;35:989-93.

8. Campo A, Mathai SC, Le Pavec J, Zaiman AL, Hummers LK, Boyce D, et al. Hemodynamic predictors of survival in scleroderma-related pulmonary arterial hypertension. Am J Respir Crit Care Med 2010;182:252-60.

9. Peacock AJ, Murphy NF, McMurray JJ, Caballero L, Stewart S. An epidemiological study of pulmonary arterial hypertension. Eur Respir J 2007;30:104-9.

10. D'Alonzo GE, Barst RJ, Ayres SM, Bergofsky EH, Brundage BH, Detre KM, et al. Survival in patients with primary pulmonary hypertension. Results from a national prospective registry. Ann Intern Med 1991;115:343-9.

11. Barst RJ, Galie N, Naeije R, Simonneau G, Jeffs R, Arneson C, et al. Long-term outcome in pulmonary arterial hypertension patients treated with subcutaneous treprostinil. Eur Respir J 2006;28:1195-203.

12. McLaughlin VV, Sitbon O, Badesch DB, Barst RJ, Black C, Galie $\mathrm{N}$, et al. Survival with first-line bosentan in patients with primary pulmonary hypertension. Eur Respir J 2005;25:244-9.

13. Welsh CH, Hassell KL, Badesch DB, Kressin DC, Marlar RA. Coagulation and fibrinolytic profiles in patients with severe pulmonary hypertension. Chest 1996;110:710-7.

14. Barst RJ, Gibbs JS, Ghofrani HA, Hoeper MM, McLaughlin VV, Rubin LJ, et al. Updated evidence-based treatment algorithm in pulmonary arterial hypertension. J Am Coll Cardiol 2009;54 (1 Suppl):S78-84.

15. Johnson SR, Granton JT, Mehta S. Thrombotic arteriopathy and anticoagulation in pulmonary hypertension. Chest 2006;130:545-52.

16. Johnson SR, Mehta S, Granton JT. Anticoagulation in pulmonary arterial hypertension: A qualitative systematic review. Eur Respir J 2006;28:999-1004.

17. Fuster V, Steele PM, Edwards WD, Gersh BJ, McGoon MD, Frye RL. Primary pulmonary hypertension: Natural history and the importance of thrombosis. Circulation 1984;70:580-7.

18. Roman A, Rodes-Cabau J, Lara B, Bravo C, Monforte V, Pallissa E, et al. [Clinico-hemodynamic study and treatment of 44 patients with primary pulmonary hypertension]. Med Clin (Barc) 2002;118:761-6.

19. Kawut SM, Horn EM, Berekashvili KK, Garofano RP, Goldsmith

Personal non-commercial use only. The Journal of Rheumatology Copyright $\odot$ 2012. All rights reserved. 
RL, Widlitz AC, et al. New predictors of outcome in idiopathic pulmonary arterial hypertension. Am J Cardiol 2005;95:199-203.

20. Ogata M, Ohe M, Shirato K, Takishima T. Effects of a combination therapy of anticoagulant and vasodilator on the long-term prognosis of primary pulmonary hypertension. Jpn Circ J 1993;57:63-9.

21. Rich S, Kaufmann E, Levy PS. The effect of high doses of calcium-channel blockers on survival in primary pulmonary hypertension. N Engl J Med 1992;327:76-81.

22. Storstein O, Efskind L, Muller C, Rokseth R, Sander S. Primary pulmonary hypertension with emphasis on its etiology and treatment. Acta Med Scand 1966;179:197-212.

23. Frank H, Mlczoch J, Huber K, Schuster E, Gurtner HP, Kneussl M. The effect of anticoagulant therapy in primary and anorectic drug-induced pulmonary hypertension. Chest 1997;112:714-21.

24. Goodwin JF, Harrison CV, Wilcken DE. Obliterative pulmonary hypertension and thrombo-embolism. Br Med J 1963;1:701-11.

25. Goodwin JF, Harrison CV, Wilcken DE. Obliterative pulmonary hypertension and thromboembolism. Br Med J 1963;1:777-83.

26. Galie N, Hoeper MM, Humbert M, Torbicki A, Vachiery JL, Barbera JA, et al. Guidelines for the diagnosis and treatment of pulmonary hypertension: the Task Force for the Diagnosis and Treatment of Pulmonary Hypertension of the European Society of Cardiology (ESC) and the European Respiratory Society (ERS), endorsed by the International Society of Heart and Lung Transplantation (ISHLT). Eur Heart J 2009;30:2493-537.

27. McLaughlin VV, Archer SL, Badesch DB, Barst RJ, Farber HW, Lindner JR, et al. ACCF/AHA 2009 expert consensus document on pulmonary hypertension: A report of the American College of Cardiology Foundation Task Force on Expert Consensus Documents and the American Heart Association developed in collaboration with the American College of Chest Physicians; American Thoracic Society, Inc.; and the Pulmonary Hypertension Association. J Am Coll Cardiol 2009;53:1573-619.

28. Beyth RJ, Quinn LM, Landefeld CS. Prospective evaluation of an index for predicting the risk of major bleeding in outpatients treated with warfarin. Am J Med 1998;105:91-9.

29. McMahan DA, Smith DM, Carey MA, Zhou XH. Risk of major hemorrhage for outpatients treated with warfarin. J Gen Intern Med 1998;13:311-6.

30. Duchini A, Sessoms SL. Gastrointestinal hemorrhage in patients with systemic sclerosis and CREST syndrome. Am J Gastroenterol 1998;93:1453-6.

31. Omair MA, Johnson SR. Gastric antral vascular ectasia unmasked by alprostadil for digital ulceration in scleroderma. J Rheumatol 2011;38:784-5.
32. Channick RN, Simonneau G, Sitbon O, Robbins IM, Frost A, Tapson VF, et al. Effects of the dual endothelin-receptor antagonist bosentan in patients with pulmonary hypertension: A randomised placebo-controlled study. Lancet 2001;358:1119-23.

33. Rubin LJ, Badesch DB, Barst RJ, Galie N, Black CM, Keogh A, et al. Bosentan therapy for pulmonary arterial hypertension. N Engl J Med 2002;346:896-903.

34. Simonneau G, Robbins IM, Beghetti M, Channick RN, Delcroix M, Denton CP, et al. Updated clinical classification of pulmonary hypertension. J Am Coll Cardiol 2009;54(1 Suppl):S43-54.

35. Johnson SR, Granton JT, Tomlinson GA, Grosbein HA, Hawker GA, Feldman BM. Effect of warfarin on survival in scleroderma-associated pulmonary arterial hypertension (SSc-PAH) and idiopathic PAH. Belief elicitation for Bayesian priors. J Rheumatol 2011;38:462-9.

36. Austin PC. Balance diagnostics for comparing the distribution of baseline covariates between treatment groups in propensity-score matched samples. Stat Med 2009;28:3083-107.

37. Spiegelhalter D, Abrams KR, Myles JP. Bayesian approaches to clinical trials and health care evaluation. Chichester: John Wiley and Sons Ltd.; 2004.

38. Sung L, Hayden J, Greenberg ML, Koren G, Feldman BM, Tomlinson GA. Seven items were identified for inclusion when reporting a Bayesian analysis of a clinical study. J Clin Epidemiol 2005;58:261-8.

39. Burton PR, Gurrin LC, Campbell MJ. Clinical significance not statistical significance: A simple Bayesian alternative to $\mathrm{p}$ values. J Epidemiol Community Health 1998;52:318-23.

40. Johnson SR, Feldman BM, Pope JE, Tomlinson GA. Shifting our thinking about uncommon disease trials: The case of methotrexate in scleroderma. J Rheumatol 2009;36:323-9.

41. Chaloner K, Rhame FS. Quantifying and documenting prior beliefs in clinical trials. Stat Med 2001;581-600.

42. Bjornsson J, Edwards WD. Primary pulmonary hypertension: A histopathologic study of 80 cases. Mayo Clin Proc 1985;60:16-25.

43. Farber HW, Loscalzo J. Pulmonary arterial hypertension. N Engl J Med 2004;351:1655-65.

44. Badesch DB, Abman SH, Ahearn GS, Barst RJ, McCrory DC, Simonneau G, et al. Medical therapy for pulmonary arterial hypertension: ACCP evidence-based clinical practice guidelines. Chest 2004;126(1 Suppl):35S-62S. 